\title{
Novel insights into biomarkers associated with renal cell carcinoma
}

\author{
BINGHAI CHEN $^{1}$, ZHIMIN JIAO $^{1}$, XIFENG YIN $^{1}$, ZHOUNAN QIAN $^{1}$, JIE GU $^{2}$ and HAO SUN ${ }^{1}$ \\ ${ }^{1}$ Department of Urology, The Affiliated Hospital of Jiangsu University; \\ ${ }^{2}$ Institute of Life Sciences, Jiangsu University, Zhenjiang, Jiangsu 212000, P.R. China
}

Received March 29, 2017; Accepted December 13, 2017

DOI: $10.3892 / \mathrm{ol} .2018 .8665$

\begin{abstract}
Renal cell carcinoma (RCC) is a common form of cancer of the urinary tract. The present study aimed to identify driver genes in RCC using a bioinformatics approach. GSE53757 and GSE40435 microarray data were analyzed, and differentially expressed genes were filtered prior to gene ontology (GO) and pathway analysis. A protein-protein interaction (PPI) network was established. Overall survival and recurrence were investigated and based on data presented in cBioPortal. The COPS7B gene within the PPI network was selected for further study in vitro. The present study identified 174 and 149 genes possessing a significant signal to noise ratio in GSE53757 and GSE40435, respectively. In total, 53 of these genes were selected based upon inclusion in both datasets. GO analysis indicated that PRKCDBP, EHD2, KCNJ10, ATP1A1, KCNJ1 and EHD2 may be involved in various biological processes. Furthermore, ALDH6A1, LDHA, SUCLG1 and ABAT may be involved in the propanoate metabolism pathway. A network consisting of 106 genes, and one typical cluster were constructed. In addition, COPS7B was selected, as it was associated with decreased overall survival and increased recurrence rates, in order to elucidate its function in RCC. Furthermore, upregulation of COPS7B was demonstrated to be predictive of advanced stage disease and metastasis of RCC. Finally, COPS7B-knockdown inhibited RCC cell proliferation and invasion ability. Collectively, these results provided novel insights into COPS7B function, indicating that COPS7B may serve as a prognostic marker and therapeutic target in RCC.
\end{abstract}

Correspondence to: Dr Jie Gu, Institute of Life Sciences, Jiangsu University, 301 Xuefu Road, Zhenjiang, Jiangsu 212000, P.R. China E-mail: 1000004531@ujs.edu.com

Abbreviations: RCC, renal cell carcinoma; GEO, gene expression omnibus; GO, gene ontology; KEGG, Kyoto encyclopedia of genes and genomes; CSN, COP9 signalosome

Key words: RCC, COP9 signalosome subunit 7B, protein-protein interaction, cBioportal, metastasis

\section{Introduction}

Renal cell carcinoma (RCC) is a common cancer of the genitourinary tract. Previous research suggests that RCC is responsible for $\sim 3 \%$ of all adult tumors, and its frequency and associated mortality rates are increasing worldwide over the past 8 years $(1,2)$. Typically, patients present with advanced-stage RCC at the time of diagnosis due to the insidious onset of RCC, which accounts for the associated poor prognosis, and the high morbidity and mortality rates of RCC (3). Increasing resistance to targeted therapies is a common occurrence in advanced RCC (4). Resistance to targeted therapy may be mediated by several mechanisms including dysregulation of genes, angiogenesis and decreased intake of TKI inhibitors by cancer cells (5). Thus, there is an urgent requirement to identify effective biomolecular markers to assist in early diagnosis and provide prognosistic information for RCC, as these do not currently exist in clinical practice.

High-throughput sequencing has been widely used to generate mass data, particularly in cancer. For example, the cBioPortal database $(6,7)$, which includes vast quantities of clinical samples and sequence data, provides visible, free-access and clear genomics datasets. In the present study, a series of long non-coding (lnc)RNAs associated with poor prognosis for patients with breast cancer was elucidated. Following the selection of one lncRNA for further study, it was identified that a specific knockout significantly inhibited cell proliferation in breast cancer (8). Previous bioinformatic analyses suggested certain genes involved in tumorigenesis; for example, by using a bioinformatic approach to study ovarian cancer, researchers identified key genes, including CCNB1, CENPF, KIF11, and ZWINT, and, therefore, were able to suggest potential therapeutic targets (9).

The present study compared two sets of microarray data to identify the various common genes. These genes were subsequently investigated using a bioinformatics approach, and a portable network graphic was established. A significant module of 11 hub genes was obtained from this network, prior to further investigation using the cBioPortal dataset. Finally, among these hub genes, COPS7B was identified to serve an oncogenic function and to be associated with the prognosis of RCC. 


\section{Materials and methods}

Gene expression ombnibus (GEO) database and data processing. PubMed (https://www.ncbi.nlm.nih.gov/pubmed) and the GEO database (https://www.ncbi.nlm.nih.gov/geo) were used in the present study. The datasets GSE40435 (10) and GSE53757 (11) were the most abundant sources of relevant data. The GSE40435 and GSE53757 gene expression profile datasets were downloaded from the GEO database. The GSE40435 gene expression profiles included 101 RCC tissues and 101 normal tissues. The GSE53757 gene expression profiles consisted of 60 RCC tissues and 60 normal tissues. Comparisons of the two GSE datasets were performed and the common genes were identified; the expression profiles of these common genes in GSE40435 were copied to another file. A heat map was constructed using Morpheus (https://software. broadinstitute.org/morpheus), and the metric in the heat map was set as the signal to noise (STN), where altered STN suggested significant expression alteration. The values $(>3$ or $\leq 3)$ suggest significant fold change of expressions. Class A and $\mathrm{B}$ were set as tumor and normal, respectively. The present study ran the Gene Ontology (GO) and Kyoto Encyclopedia of Genes and Genomes (KEGG) analyses in DAVID version 6.8 (http://david.abcc.ncifcrf.gov).

Gene network building and hub genes analysis. The present study included all common genes identified in the two GEO Serious (GSE) datasets (GSE40435 and GSE53757) located within the STRING database (http://string.embl.de/). A protein-protein interaction (PPI) network was constructed using Cytoscape version 3.6 (http://www.cytoscape.org). All datasets were identical to those previously reported (9).

Database search of cBioPortal. All hub genes were input and recognized in the cBioPortal (http://www.cbioportal.org/). The dataset for RCC carcinoma (TCGA, provisional) was selected as it has numerous cases, compared with other datasets. Alterations in the mutations, copy number and expression of various hub genes were analyzed. Clinical feathers, including stage, metastasis, the overall survival and disease free survival, sex, age, laterality and volume were analyzed based on the clinical data from cBioPortal. The cBioPortal also generated the Kaplan-Meier curve following input of the gene name for disease/progression-free-survival. $\mathrm{P}<0.05$ was considered to indicate a statistically significant difference.

Cell culture, regents and siRNA transfection. The Human kidney cortex proximal tubule cell line (HK-2) and 786-O, 769-P and A498 cell lines were obtained from the American Type Culture Collection (Manassas, VA, USA) and cultured in RPMI-1640 medium (Hyclone; GE Healthcare Life Sciences, Logan, UT, USA) supplemented with $10 \%$ fetal bovine serum (Gibco; Thermo Fisher Scientific, Inc., Waltham, MA, USA). and $2 \mathrm{mM}$ glutamine (Sangon Biotech Co., Ltd., Shanghai, China). Polymerase chain reaction (PCR) primers (Table I) were purchased from Sangon Biotech Co., Ltd. (Shanghai, China). The small interfering (si)RNA negative control and COPS7B siRNA at the concentration of $40 \mathrm{nM}$ were purchased from Shanghai GenePharma Co., Ltd., Shanghai, China. The siRNAs were transfected into cells using Lipofectamine ${ }^{\circledR} 2000$
(Invitrogen, Thermo Fisher Scientific, Inc., Waltham, MA, USA) for $48 \mathrm{~h}$ prior to subsequent experimentation. A random sequence (Sense: 5'-UUCUCCGAACGUGUCACGUTT-3' Antisense: 5'-ACGUGACACGUUCGGAGAATT-3') from Shanghai GenePharma Co., Ltd. was used as the negative control.

Reverse transcription-quantitative $(R T-q) P C R$. Total RNA of RCC tissue and cell lines were extracted with TRIzol ${ }^{\circledR}$ reagent (Thermo Fisher Scientific, Waltham, MA, USA.) prior to concentration determination with a Nanodrop reader (Thermo Fisher Scientific, Inc.). RNA was reverse transcribed to cDNA with ReverTra Ace- $\alpha-{ }^{\circledR}$ (Toyobo, Osaka, Japan). The RT-qPCR was performed with Brilliant SYBR Green Master mix (Bio-Rad Laboratories, Hercules, CA, USA) on a Roche LightCycler ${ }^{\circledR} 480$ Instrument II (Roche Applied Science, Mannheim, Germany) according to the study of Liu et al (8). All primers are listed in Table I. The thermocycling conditions were as follows: $95^{\circ} \mathrm{C}$ for $5 \mathrm{~min}, 95^{\circ} \mathrm{C}$ for $10 \mathrm{sec}$ and $60^{\circ} \mathrm{C}$ for $30 \mathrm{sec}$ ( 40 cycles), $95^{\circ} \mathrm{C}$ for $15 \mathrm{sec}, 60^{\circ} \mathrm{C}$ for $60 \mathrm{sec}$ and $95^{\circ} \mathrm{C}$ for $15 \mathrm{sec}$.

Cell survival assay. The $769-\mathrm{P}$ cells $\left(2 \times 10^{4}\right)$ were seeded in 12-well plates and cultured overnight following transfection with siRNA-COP9 signalosome subunit 7B (COPS7B) or siRNA control for $48 \mathrm{~h}$. In total, $10 \mu \mathrm{l}$ Cell Counting Kit-8 (Dojindo, Rockville, MD, USA) was added after 24, 48 and 72 h. Optical density (OD) values were determined based on the absorbance at $450 \mathrm{~nm}$ with Nanodrop reader. Cell survival was calculated according to the following formula: $\left[\mathrm{OD}_{\text {(time) }}-\mathrm{OD}_{\text {(blank) }}\right] /\left[\mathrm{OD}_{(0 \mathrm{~h})}-\mathrm{OD}_{\text {(blank })}\right]$.

Colony formation. Following transfection with the siRNA control or si-COPS7B at $37^{\circ} \mathrm{C}$ for $48 \mathrm{~h}, 2,000$ cells/well were seeded in 6-well plates. At 2 weeks after seeding, colonies were fixed and stained with $0.1 \%$ crystal violet at room temperature for $10 \mathrm{~min}$. Finally, all the colonies were counted with the naked eye, and then colony-forming efficiency was calculated as described previously (8).

Cell migration assay. Following transfection with the siRNA control or si-COPS7B at $37^{\circ} \mathrm{C}$ for $48 \mathrm{~h}, 769-\mathrm{P}$ cells were starved for $3 \mathrm{~h}$ prior to cell counting. In total, $1 \times 10^{4}$ cells in serum-free medium were seeded into the upper chambers of a BioCoat invasion system from BD Biosciences (San Jose, CA, USA) and completed RPMI-1640 was placed in the lower chamber. Then it was incubated at $37^{\circ} \mathrm{C}$ and $5 \% \mathrm{CO}_{2}$ overnight. After $24 \mathrm{~h}$, cells in the upper membrane were wiped away with a cotton pad. The cells on the lower surface of the membrane were stained with crystal violet at a concentration of $0.1 \%$ at room temperature for $30 \mathrm{~min}$. The stained 769-P cells were counted and the mean cell numbers were calculated in five fields with light microscope at x40 magnification (12).

Statistical analysis. Statistical analysis was performed using SPSS software (version 17; SPSS Inc., Chicago, IL, USA). One-way analysis of variance was conducted, with the Tamhane's T2 post hoc test to consider heterogeneity of variance. Data are expressed as the mean \pm standard error of the mean. Data were analyzed using the Student's t-test. The log 
Table I. Primers in the present study.

\begin{tabular}{lll}
\hline Gene & \multicolumn{1}{c}{ Sense } & \multicolumn{1}{c}{ Antisense } \\
\hline CA9 & AGGTGAATGCAGAGGTGACA & AAGCTGGAGAGAGAGGGAGA \\
NDUFA4L2 & AAAAGTTGGCATGGCAGGAG & GCTCCGGGTTGTTCTTTCTG \\
HRG & CTTCTACCACCAAGCCTCCA & GCAGGACATGGGCAATAGTG \\
KCNJ1 & TCTTCGGAAATGGGTCGTCA & CTGCATACCACAGGAGACCA \\
KNG1 & GGTTGGCTCTGACACGTTTT & TGGGTAGCCACGGAGAATTT \\
UMOD & GTTTTCATCCCGCAGAGCAA & ATCGCCCGGTTAAATGTCG \\
COPS7 & AAAGACCTGGAGATGCGGAA & AGCCATCACACCATTCATGC \\
\hline
\end{tabular}

A

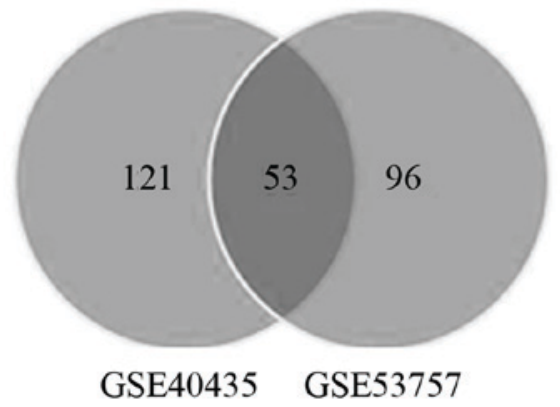

$\mathrm{C}$

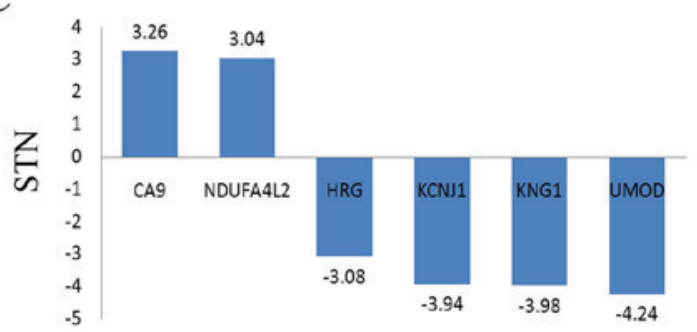

B

Cancer

Normal

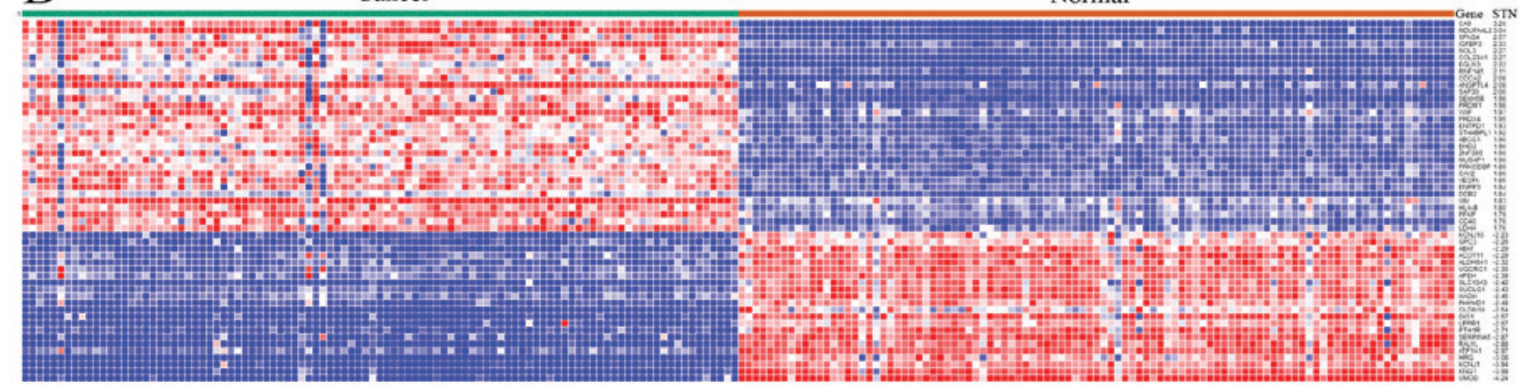

Figure 1. Identification of genetic alterations in GEO datasets. (A) Identification of common genes expressed in GSE53757 and GSE40435 datasets. (B) Heat map of 53 genes expressed in the GSE53757 and GSE40435 datasets. GEO, Gene Expression Omnibus. (C) Six typical genes with significant STN. RCC, renal cell carcinoma.

rank test and Kaplan-Meier were conducted to determinate overall survival and recurrence. P-values were two-sided, and $\mathrm{P}<0.05$ was considered to indicate a statistically significant difference.

\section{Results}

Identification of genetic alterations and analysis of functions in GEO datasets. To acquire sufficient data from the gene microarrays, two GEO chip files, GSE53757 and GSE40435 were downloaded. The present study identified 174 and 149 genes that have significant STN ratios in the GSE53757 and GSE40435 datasets, respectively. Following comparisons between these two datasets, 53 of the genes were selected as they featured in both sets (Fig. 1A). All data associated with these 53 genes (available upon request) were input to Morpheus (https://software.broadinstitute.org) and a new heat map was constructed (Fig. 1B). Among these genes, carbonic anhydrase 9 (CA9) and NDUFA4L2 possessed an
STN ratio of $>3$, whereas $\mathrm{HRG}, \mathrm{KCNJ} 1, \mathrm{KNG} 1$ and UMOD had an STN ratio of <-3 (Fig. 1C). Furthermore, to investigate the gene function(s) and the potential pathways involved, the present study analyzed the data using DAVID $(13,14)$, in which GO and KEGG analysis were conducted. All 53 genes were recognized by DAVID. GO analysis indicated that PRKCDBP, EHD2KCNJ10, ATP1A1, KCNJ1 and EHD2 may be involved in the process of cortical actin cytoskeleton organization, potassium-ion import, positive regulation of endocytic recycling and membrane tabulation, respectively. In addition, ALDH6A1, LDHA, SUCLG1 and ABAT may be involved in the propanoate metabolism pathway.

Analysis of hub genes in RCC. To elucidate the critical hub genes in RCC, the STRING database was used to build a portable network graphic which consisted of 106 genes. As presented in Fig. 2A, a cluster of genes was identified to be markedly associated with other genes. It included DDB2, COPS6, DDB1, COPS5, COPS7A, COPS8, COPS4, GPS1, 


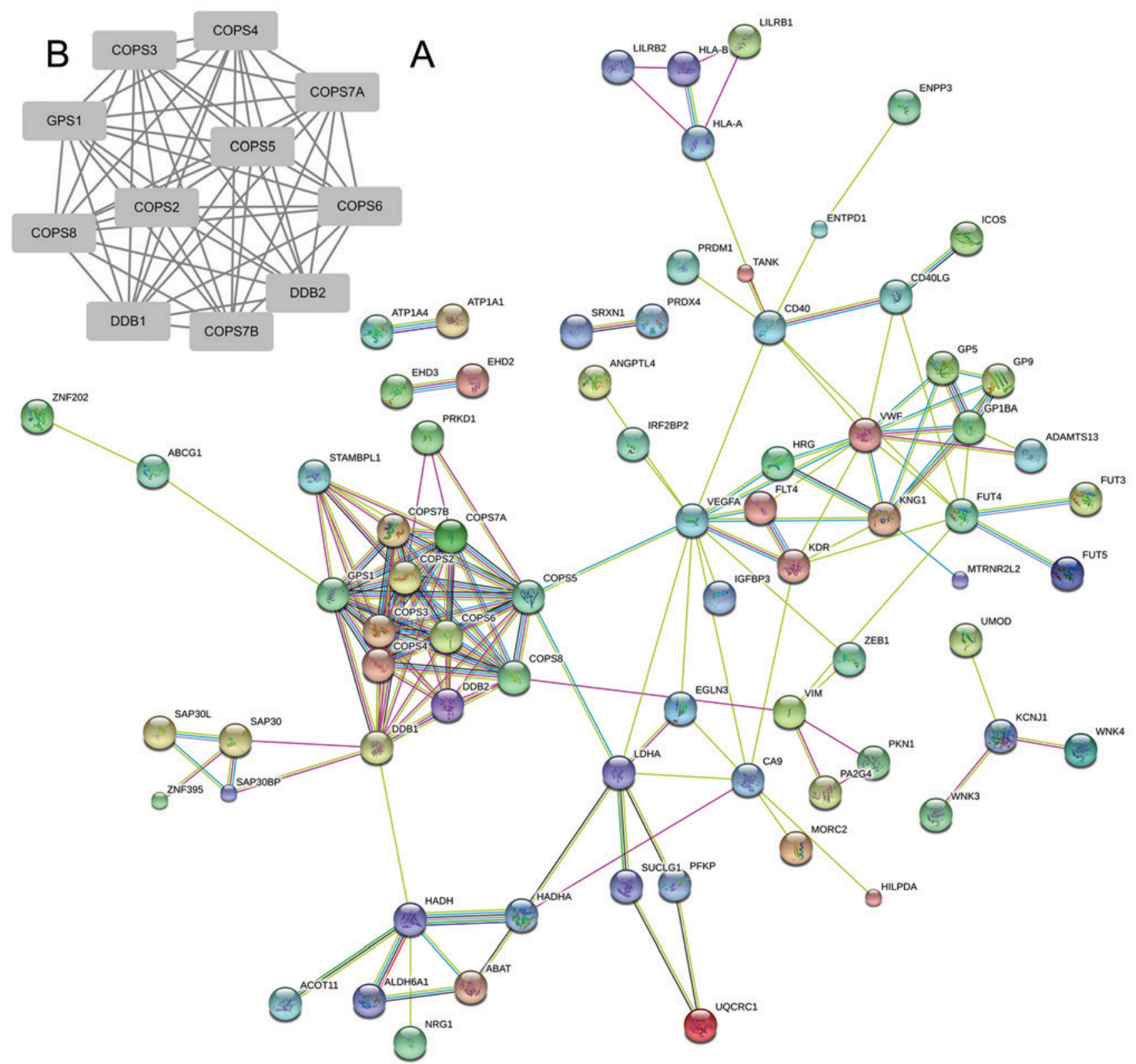

Figure 2. Analysis of hub genes in RCC. (A) Protein-protein interaction network of differentially expressed genes in RCC. (B) A representative module picked up from the network. The lines represent associations between proteins.

COPS3 COPS7B and COPS2. Among these genes, eight are members of the COP9 family; the remaining two are members of the DDB family. These results suggest that both COP9 and DDB may be crucial in the detection of RCC. To further validate the hub genes in RCC, Cytoscape was used to construct a PPI network (15). A significant module was obtained from the network using MCODE in Cytoscape, which included 11 nodes (Fig. 2B). These results revealed the crucial role of the COP9 family in this module; these were also similar to the results derived from STRING analysis. Notably, GPS1 was associated with all other genes in the module, which may provide direction for further study in RCC.

Hub genes are significantly associated with overall survival and recurrence in $R C C$. The 11 genes specified in Fig. 2B were analyzed using the $\mathrm{cBioPortal}$ database, which contained 538 clinical cases of RCC and sequenced data. As presented in the cBioPortal database, genetic alterations are comprised of three parameters: Mutation, Copy Number Alteration (CNA) and expression. The present study focused on the expression of the hub genes. The significant associations of COPS7B with overall survival and recurrence were observed using a Kaplan-Meier curve. The log rank test P-values for these are $2.926 \times 10^{-5}$ and 0.021 , respectively, (Fig. 3A and B). No other hub genes were associated with overall survival and disease free survival. These results indicate that the COPS7B may be important in monitoring the development of RCC and also serve as a valuable biological marker.

Clinical features of COPS7B in cBioPortal. To reveal the clinical significance of COPS7B in RCC, genetic alterations in COPS7B, as well as alterations to other clinical features (information available upon request), were investigated. Notably, COPS7B was identified to be associated with clinical stage 

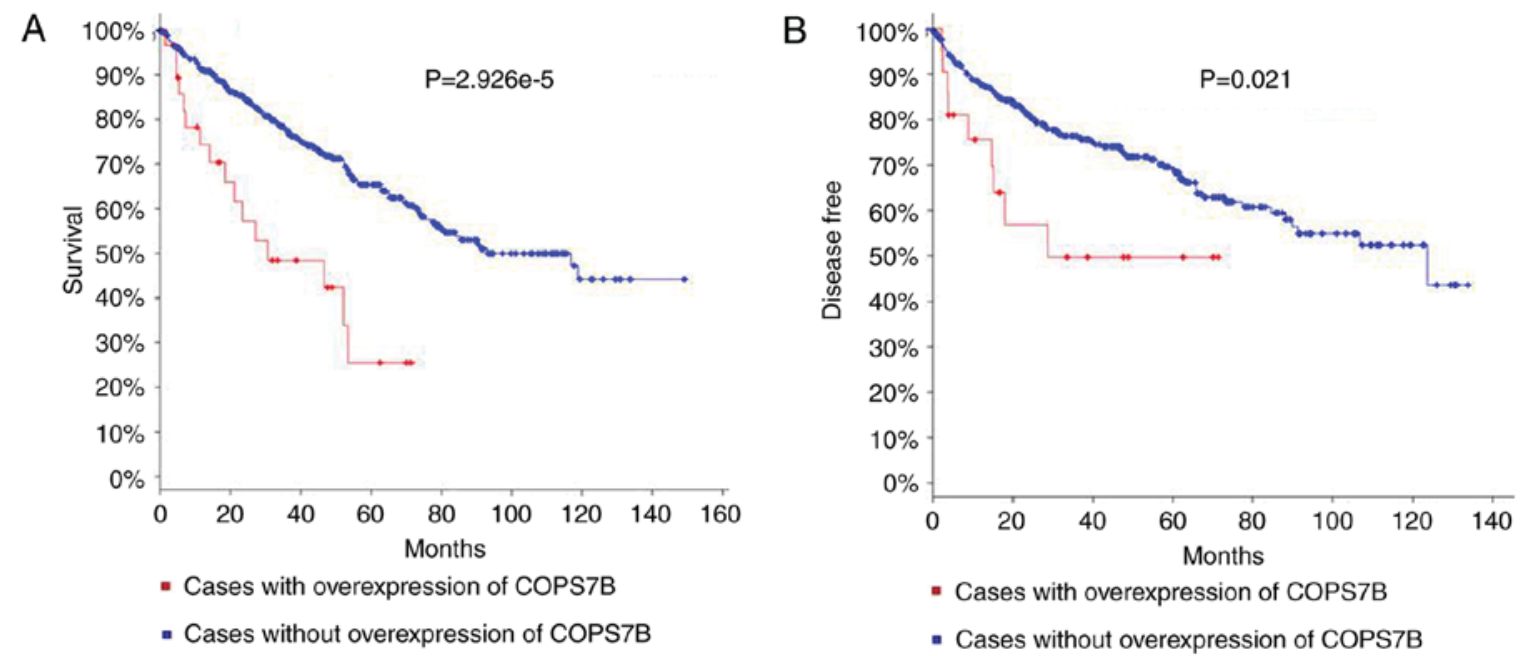

Figure 3. Hub gene COPS7B predicts overall survival and disease-free survival (months) in renal cell carcinoma. (A) Kaplan-Meier curve for overall survival according to alterations in COPS7B. (B) Kaplan-Meier curve for disease-free survival (months) according to alterations of COPS7B. COPS7B, COP9 signalosome subunit 7B.

and metastasis. As presented in Fig. 4A, $49 \%$ of cases with COPS7B overexpression were diagnosed with stage IV RCC. On the other hand, $24 \%$ of cases with normal expression of COPS7B were stage IV, suggesting that COPS7B may be associated with advanced-stage IV RCC (Fig. 4A). Furthermore, metastasis was identified in $31 \%$ of cases in which COPS7B was overexpressed, while $14 \%$ presented with metastasis without concomitant COPS7B overexpression (Fig. 4B). Other clinical features, including sex, age, laterality and volume, were evaluated separately, and no significant associations with COPS7B were observed.

Knockdown of COPS7B by siRNA in RCC cell lines. The present study demonstrated the overexpression of COPS7B is associated with poor overall survival, recurrence, advanced stage and metastasis. Based on the data provided by the GEO chip file GSE40435 (203 tissues), the expression of COPS7B was determined. It indicated that the COPS7B was overexpressed in RCC compared with in adjacent normal tissues (Fig. 5A). Furthermore, these results indicated that COPS7B may function as an oncogene in RCC. To confirm this function, we focused on the level of COPS7B expression in various RCC cell lines (786-O, 769-P and A498) as well as in normal renal tubular epithelial HK-2 cells. The results demonstrated that COPS7B was overexpressed in 769-P cells, compared with in HK-2 cells (Fig. 5B). Furthermore, we then used siRNA to knock-down COPS7B in 769-P cells (Fig. 5C).

Oncogenic role of $C O P S 7 B$ in $R C C$. Cell survival assays suggested that the knock-down of COPS7B inhibited cell proliferation $48 \mathrm{~h}$ following transfection, as compared with the siRNA control (Fig. 5D). To confirm that knock-down of COPS7B inhibits cell proliferation, colony formation was assessed following transfection, with effective siRNA knockdown (Fig. 5E). Considering that COPS7B is associated with metastasis, as determined based on the data from cBioPortal, an invasion assay was performed following COPS7B knockdown. The invasion assay demonstrated that knockdown of
A

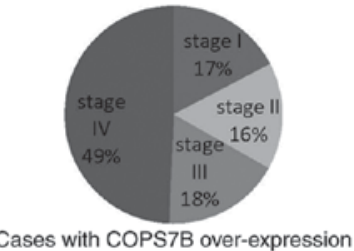
$\mathrm{n}=26$

B

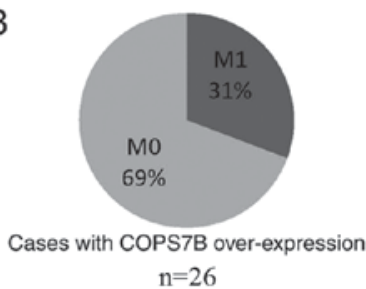
Cases without COPS7B over-expression $n=494$

Figure 4. Clinical features of the expression of COPS7B in renal cell carcinoma. (A) Cancer stage distribution in cases with $(n=26)$ and without $(n=494)$ overexpressed COPS7B. (B) Percentage of metastasis in cases with $(n=26)$ and without $(n=494)$ overexpressed COPS7B. M0, no metastasis; M1, metastasis; Staging classification: American Joint Committee on Cancer pathologic tumor stage.

COPS7B inhibited the invasion ability of 769-P cells, indicating the oncogenic function of COPS7B in RCC (Fig. 5F).

\section{Discussion}

A previous study investigated the molecular markers of RCC; however, little is known regarding the clinical significance of any specific marker (16). The present study focused on two microarray datasets and used a series of biological software investigations to identify hub genes. The present study also screened the hub genes in cBioPortal, a database containing a large amount of sequencing data, as well as clinical features that may be associated with RCC. Thus, these hub genes were characterized as follows: 1) Hub genes were generated based on expression alterations in 161 tumors and 161 tumor adjacent tissues; 2) hub genes were screened in the cBioPortal with 538 RCC cases, and COPS7B was associated with prognosis; 3) 


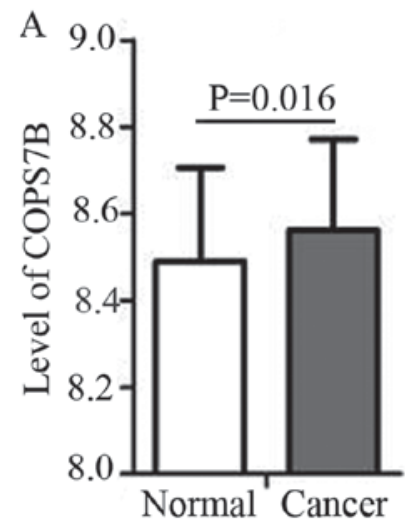

D

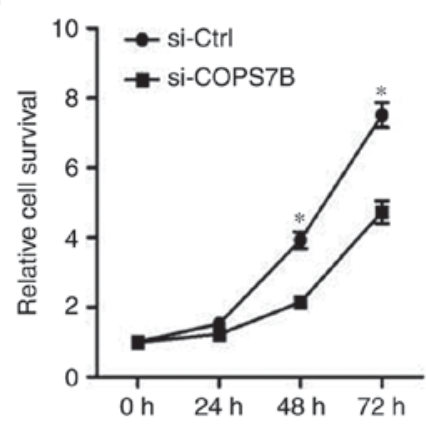

B

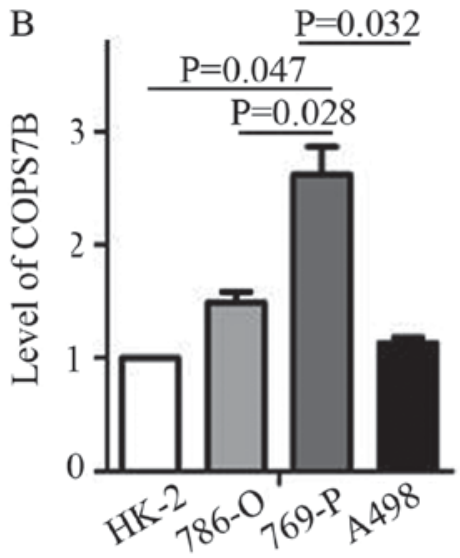

E

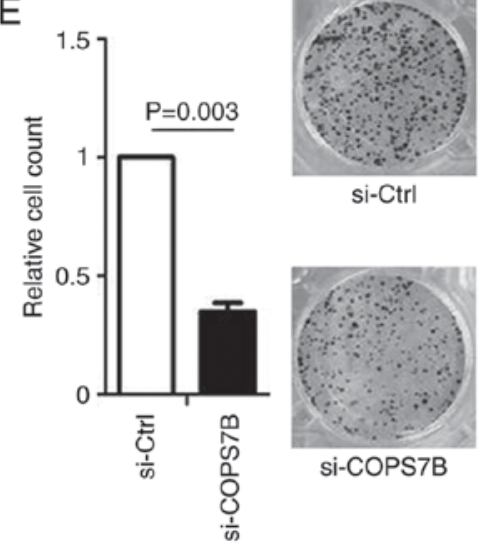

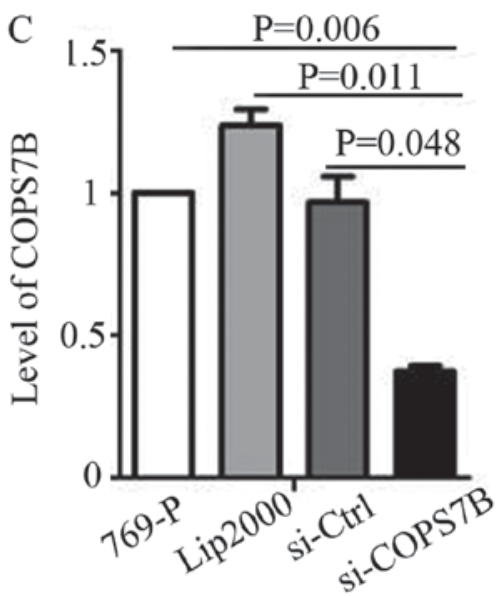

$\mathrm{F}$

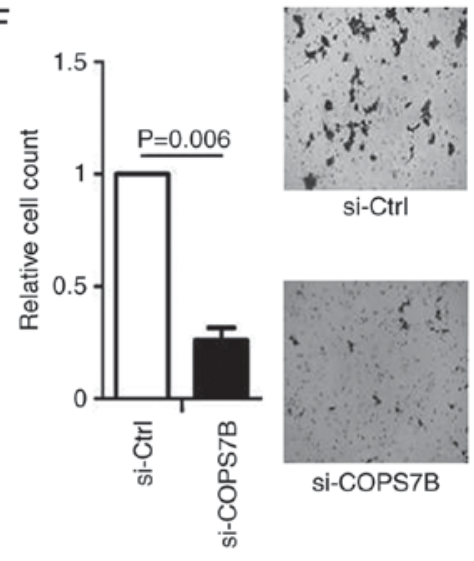

Figure 5. Oncogenic role of COPS7B in vivo and in vitro. (A) An in vivo experiment presenting the altered expression of COPS7B in the GSE40435 dataset. (B) Overexpression of COPS7B in renal cell carcinoma cell lines, particularly in 769-P cells in vitro. (C) Knock-down of COPS7B by siRNA in 769-P cells. (D) si-COPS7B inhibits 769-P cell survival in vitro. (E) si-COPS7B inhibits colony formation of 769-P cells in vitro. (F) si-COPS7B inhibits the cell invasion of 769-P cells in vitro. ${ }^{*} \mathrm{P}<0.05$ vs. si-COPS7B. COPS7B, COP9 signalosome subunit $7 \mathrm{~B}$; Ctrl, control.

The hub gene COPS7B is predictive of overall survival and recurrence for patients with $\mathrm{RCC}$. These results suggest that COPS7B is a potential prognostic marker in RCC and warrants further investigation.

A previous study investigating molecular markers to determine prognosis in RCC focused on VHL, hypoxia inducible factor 1- $\alpha$ (HIF1- $\alpha$ ), VEGF, CA9 and Ki-67 (16). The results of a previous study demonstrated that VHL is a tumor suppressor gene in RCC. Its product, phosphorylated-VHL, is relevant to numerous cell-signaling pathways including the HIF1- $\alpha$ pathway, and regulates oxidative phosphorylation. The inactivation of VHL leads to aberrant VHL-HIF-VEGF pathway activation associated with oncogenesis and tumor development in RCC (17). In the GSE40435 dataset, the present study surveyed VHL expression in 202 samples. It was identified that VHL was overexpressed in tumors, a result consistent with those of other studies. However, VHL does not meet the criteria for a hub gene. This is may be due to several reasons, including variation in the detection methods of VHL between datasets. Furthermore, VHL did not meet the requirements when constructing the visual molecular interaction networks (Cytoscape). Therefore, COPS7B may be more important as a hub gene, compared with VHL.

Additionally, previous study suggests that several genes in the serum were indicated to be non-invasive and associated with metastasis, recurrence and prognosis. CA9 was over-expressed in RCC compared with in normal renal samples (18). CA9 expression, which is detectable in serum, has previously been reported to be associated with prognosis due to early-activated HIF. However, the present study observed that COPS7B is a novel molecular marker, based on analysis of a large number of clinical cases, suggesting that COPS7B may serve as a clinical biomarker for RCC. Currently, little is known about biomarkers in the urine. The expression of NMP22 in the urine of patients with RCC was significantly increased, compared with the controls. A previous study reported that urinary NMP22 may, therefore, allow the early detection of RCC (19). However, due to the low positive test rate, the clinical significance of NMP22 requires further evaluation. The potential oncogenic gene COPS7B may offer an alternative and promising diagnosis strategy for RCC by analyzing its expression levels in urine.

This bioinformatic study provides new perspectives to identify novel biomarkers in RCC. Future investigations analyzing additional online datasets will allow screening for other potential biomarkers for RCC. For example, data from the metastasis dataset provides a number of genes associated with metastasis (20). In addition, there are various clinical stage-associated genes, including ephA2, in the datasets that warrant further study (21). Thus, biomarkers for different 
biological functions may be uncovered based on these microarray results. A previous study on ovarian cancer screened out 345 genes and 36 differentially expressed miRNAs, suggesting that a number of genes and miRNAs may serve crucial functions in ovarian carcinoma (9). Compared with the findings of this previous study, the present study focused on the hub genes that were identified in the cBioPortal database (538 clinical cases) to reveal the role of the hub genes in overall survival and disease recurrence. Furthermore, the present study validated the oncogenic function in various RCC cell lines. Collectively, these studies suggest that online data analysis is an effective tool in the evaluation of cancer prognosis and identifying potential therapeutic targets. Furthermore, it is possible to identify the underlying molecular mechanisms using online software. For example, COPS7B was analyzed using the cBioPortal, identifying the co-expression of COPS7B and ATG4B, with a Pearson correlation of up to 0.79 , therefore indicating that COPS7B is closely associated with ATG4B. Thus, ATG4B may be upstream or downstream of COPS7B. Similarly, other genes associated with COPS7B must be identified prior to elucidating the mechanisms.

CSN7 is an important subunit of COP9 signalosome (CSN), which is a conserved protein complex that includes 8 CSNs. CSN7 includes the COPS7A and COPS7B complexes, and is one of the most prominent phosphorylated subunits formed in response to DNA damage $(22,23)$. CSN has notable effects on multiple cell signaling functions, including DNA repair, cell cycle control, angiogenesis and tumor development (24). An increase in the CSN holo-complex under cancerous conditions may lead to the development of drug resistance (25).

Furthermore, COPS7A may be considered a biomarker predicting recurrence and prognosis of RCC. Considering the important roles of CSN in cancer, it is likely that COPS7B may also have important roles in other solid tumors. Additionally, the knockdown of COPS7B inhibited HIF1- $\alpha$ (information available upon request). Thus, the inhibition of HIF1- $\alpha$ following COPS7B knockdown may be a mechanism of COPS7B in RCC. The mechanisms associated with this phenotype in RCC have not been clearly demonstrated in the present study; however, it is possible that HIF1- $\alpha$ may be involved downstream, thus warranting further investigation.

It is also reported that COPS7A and COPS7B complexes co-exist in human red blood cells (26). The results of the present study suggest that COPS7A and COPS7B may also coexist and exert synergistic effects in RCC. These synergistic effects may be based on the spatial structure of the two proteins, which are similar to the miRNA previously reported (27). Further investigation of the COPS7A and COPS7B complexes may explain the role of COPS7B suggested by the present study and assist in identifying novel targets for RCC therapy. Although the role and underlying mechanism of COPS7B in RCC remains to be determined, its association with overall survival and disease recurrence is supported by the results of the present study, which indicate that COPS7B has an oncogenic role. Considering that COPS7B is regarded as a hub gene, it is hypothesized that COPS7B may be a driver gene and serve as a biological marker as well as a therapeutic target in RCC.

The present study provided an example of an integrated bioinformatics analysis approach to identify potential biomarkers, hub genes for diagnosis and for the prediction of overall survival, disease recurrence and therapeutic targets. Due to the extensive clinical chip data now available online, the present study demonstrated how researchers may utilize a bioinformatics approach to discover genes that serve key functions in cancer. Specifically, the cBioPortal encompasses genomic data from 105 types of cancer, and its expansion may provide new research opportunities as well as save time and resources.

\section{Acknowledgements}

Not applicable.

\section{Funding}

The present study was supported by the National Natural Science Foundation of China (grant nos. 81402100 and 31600952), the Foundation of Health and Family Planning Commission of Jiangsu Province (grant no. Q201408), the Social Development Foundation of Zhenjiang (grant nos. SH2016031 and SH2014026), the foundation from the Foundation for Jiangsu Provincial Medical Youth Talent (grant no. QNRC2016840), and the Six Talent Peaks Project of Jiangsu Province (grant no. WSW-007).

\section{Availability of data and materials}

The datasets used and/or analyzed during the current study are available from the corresponding author on reasonable request.

\section{Authors' contributions}

BC took responsibility for the majority of the present study, including PCR experimentation, data analysis and manuscript preparation. ZJ, XY, ZQ, JG and HS participated in study design, helped to draft the manuscript and contributed intellectually. JG provided specific academic guidance on experimental design, assistance in data analysis and review of the manuscript.

\section{Ethics approval and consent to participate}

This study was approved by the Ethics Committee of the Affiliated Hospital of Jiangsu University.

\section{Consent for publication}

Not applicable.

\section{Competing interests}

The authors declare that they have no competing interests.

\section{References}

1. Chow WH, Dong LM and Devesa SS: Epidemiology and risk factors for kidney cancer. Nat Rev Urol 7: 245-257, 2010.

2. Patard JJ, Pignot G, Escudier B, Eisen T, Bex A, Sternberg C, Rini B, Roigas J, Choueiri T, Bukowski R, et al: ICUD-EAU international consultation on kidney cancer 2010: Treatment of metastatic disease. Eur Urol 60: 684-690, 2011. 
3. Arabsalmani M, Mohammadian-Hafshejani A, Ghoncheh M, Hadadian F, Towhidi F, Vafaee K and Salehiniya H: Incidence and mortality of kidney cancers, and human development index in Asia; a matter of concern. J Nephropathol 6: 30-42, 2017.

4. Waalkes S, Kramer M, Herrmann TR, Schrader AJ, Kuczyk MA and Merseburger AS: Present state of target therapy for disseminated renal cell carcinoma. Immunotherapy 2: 393-398, 2010.

5. Duran I, Lambea J, Maroto P, González-Larriba JL, Flores L, Granados-Principal S, Graupera M, Sáez B, Vivancos A and Casanovas O: Resistance to targeted therapies in renal cancer: The importance of changing the mechanism of action. Target Oncol 12: 19-35, 2017.

6. Gao J, Aksoy BA, Dogrusoz U, Dresdner G, Gross B, Sumer SO, Sun Y, Jacobsen A, Sinha R, Larsson E, et al: Integrative analysis of complex cancer genomics and clinical profiles using the cBioPortal. Sci Signal 6: pl1, 2013.

7. Cerami E, Gao J, Dogrusoz U, Gross BE, Sumer SO, Aksoy BA, Jacobsen A, Byrne CJ, Heuer ML, Larsson E, et al: The cBio cancer genomics portal: An open platform for exploring multidimensional cancer genomics data. Cancer Discov 2: 401-404, 2012

8. Liu H, Li J, Koirala P, Ding X, Chen B, Wang Y, Wang Z, Wang C, Zhang $\mathrm{X}$ and Mo YY: Long non-coding RNAs as prognostic markers in human breast cancer. Oncotarget 7: 20584-20596, 2016.

9. Xu Z, Zhou Y, Cao Y, Dinh TL, Wan J and Zhao M: Identification of candidate biomarkers and analysis of prognostic values in ovarian cancer by integrated bioinformatics analysis. Med Oncol 33: 130, 2016.

10. von Roemeling CA, Radisky DC, Marlow LA, Cooper SJ, Grebe SK, Anastasiadis PZ, Tun HW and Copland JA: Neuronal pentraxin 2 supports clear cell renal cell carcinoma by activating the AMPA-selective glutamate receptor-4. Cancer Res 74: 4796-4810, 2014

11. Wozniak MB, Le Calvez-Kelm F, Abedi-Ardekani B, Byrnes G, Durand G, Carreira C, Michelon J, Janout V, Holcatova I, Foretova L, et al: Integrative genome-wide gene expression profiling of clear cell renal cell carcinoma in Czech Republic and in the United States. PLoS One 8: e57886, 2013.

12. Chen B, Liu J,Ho TT, Ding X and Mo YY: ERK-mediated NF-kB activation through ASIC1 in response to acidosis. Oncogenesis 5: e279, 2016

13. Huang da W, Sherman BT and Lempicki RA: Systematic and integrative analysis of large gene lists using DAVID bioinformatics resources. Nat Protoc 4: 44-57, 2009.

14. Huang da W, Sherman BT and Lempicki RA: Bioinformatics enrichment tools: Paths toward the comprehensive functional analysis of large gene lists. Nucleic Acids Res 37: 1-13, 2009.

15. Shannon P, Markiel A, Ozier O, Baliga NS, Wang JT, Ramage D, Amin N, Schwikowski B and Ideker T: Cytoscape: A software environment for integrated models of biomolecular interaction networks. Genome Res 13: 2498-2504, 2003.

16. Barata PC and Rini BI: Treatment of renal cell carcinoma: Current status and future directions. CA Cancer J Clin 67: 507-524, 2017.
17. Schonenberger D, Rajski M, Harlander S and Frew IJ: Vhl deletion in renal epithelia causes HIF-1 $\alpha$-dependent, HIF-2 $\alpha$-independent angiogenesis and constitutive diuresis. Oncotarget 7: 60971-60985, 2016.

18. Tostain J, Li G, Gentil-Perret A and Gigante M: Carbonic anhydrase 9 in clear cell renal cell carcinoma: A marker for diagnosis, prognosis and treatment. Eur J Cancer 46: 3141-3148, 2010.

19. Kaya K, Ayan S, Gokce G, Kilicarslan H, Yildiz E and Gultekin EY: Urinary nuclear matrix protein 22 for diagnosis of renal cell carcinoma. Scand J Urol Nephrol 39: 25-29, 2005

20. Jones J, Otu H, Spentzos D, Kolia S, Inan M, Beecken WD, Fellbaum C, Gu X, Joseph M, Pantuck AJ, et al: Gene signatures of progression and metastasis in renal cell cancer. Clin Cancer Res 11: 5730-5739, 2005.

21. Herrem CJ, Tatsumi T, Olson KS, Shirai K, Finke JH, Bukowski RM, Zhou M, Richmond AL, Derweesh I, Kinch MS and Storkus WJ: Expression of EphA2 is prognostic of disease-free interval and overall survival in surgically treated patients with renal cell carcinoma. Clin Cancer Res 11: 226-231, 2005.

22. Dessau M, Halimi Y, Erez T, Chomsky-Hecht O, Chamovitz DA and Hirsch JA: The Arabidopsis COP9 signalosome subunit 7 is a model PCI domain protein with subdomains involved in COP9 signalosome assembly. Plant Cell 20: 2815-2834, 2008.

23. Beli P, Lukashchuk N, Wagner SA, Weinert BT, Olsen JV, Baskcomb L, Mann M, Jackson SP and Choudhary C: Proteomic investigations reveal a role for RNA processing factor THRAP3 in the DNA damage response. Mol Cell 46: 212-225, 2012.

24. Gummlich L, Rabien A, Jung K and Dubiel W: Deregulation of the COP9 signalosome-cullin-RING ubiquitin-ligase pathway: Mechanisms and roles in urological cancers. Int J Biochem Cell Biol 45: 1327-1337, 2013.

25. Feist M, Huang X, Müller JM, Rau B and Dubiel W: Can hyperthermic intraperitoneal chemotherapy efficiency be improved by blocking the DNA repair factor COP9 signalosome? Int J Colorectal Dis 29: 673-680, 2014.

26. Rozen S, Tieri A, Ridner G, Stark AK, Schmaler T, Ben-Nissan G, Dubiel W and Sharon M: Exposing the subunit diversity within protein complexes: A mass spectrometry approach. Methods 59: 270-277, 2013.

27. Chen B, Duan L, Yin G, Tan J and Jiang X: Simultaneously expressed miR-424 and miR-381 synergistically suppress the proliferation and survival of renal cancer cells-Cdc2 activity is up-regulated by targeting WEE1. Clinics (Sao Paulo) 68: 825-833, 2013.

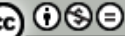

This work is licensed under a Creative Commons Attribution-NonCommercial-NoDerivatives 4.0 International (CC BY-NC-ND 4.0) License. 